\title{
Open reduction and internal fixation in temporomandibular joint traumatology: current concepts and future perspectives
}

\author{
Andreas Neff \\ Department of Oral and Craniomaxillofacial Surgery, UKGM GmbH, University Hospital Marburg and Philipps University Marburg, \\ Baldingerstrasse, Marburg D-35043, Germany.
}

Correspondence to: Prof. Andreas Neff, Department of Oral and Craniomaxillofacial Surgery, UKGM GmbH, University Hospital Marburg and Philipps University Marburg, Baldingerstrasse, Marburg D-35043, Germany. E-mail: neffa@med.uni-marburg.de

How to cite this article: Neff A. Open reduction and internal fixation in temporomandibular joint traumatology: current concepts and future perspectives. Stomatological Dis Sci2019;3:2. http://dx.doi.org/10.20517/2573-0002.2018.27

Received: 16 Dec 2018 Accepted: 22 Jan 2019 Published: 20 Feb 2019

Science Editors: Raúl González-García Copy Editor: Cai-Hong Wang Production Editor: Huan-Liang Wu

\begin{abstract}
Since the turn of the century a paradigm shift has challenged the centuries old tradition of non-surgical treatment for condylar fractures. The advent of functionally stable osteosynthesis introduced into condylar traumatology in the 1980ies step by step widened out the indications for surgical treatment, paralleled also by improvement in computerized tomography diagnostics and more recently by continuous refinement of indication-specific osteosynthesis materials, with three-dimensional-plates for the condylar base and neck and resorbable materials for the condylar head being the latest developments. Over the last decades, a series of new approaches was introduced, too, to cope with the specific demands for open reduction and rigid internal fixation (ORIF) in the different fracture locations, which according to the recent consensus based Arbeitsgemeinschaft für Osteosynthese comprehensive injury automatic classifier (AOCOIAC) classification are now defined as fractures of the condylar base, condylar neck and condylar head. By now even fractures of the condylar head, treated surgically by Rasse already in 1992, have moved into the focus of surgical interest and ORIF by now is far from being "experimental", as dubbed only a decade ago. According to recent consensus (International Bone Research Association, 2012) and based on improved evidence, ORIF may now be considered as the golden standard for both displaced or dislocated condylar base and neck fractures in adults. Endoscopic approaches should be considered for base fractures with lateral displacement. Meanwhile, there is a growing tendency to perform ORIF in condylar head fractures and severely displaced or dislocated fractures in younger children with mixed dentition.
\end{abstract}

Keywords: Condylar base, condylar neck, condylar head, open reduction and rigid internal fixation, condylar process, classification, approaches, temporomandibular joint

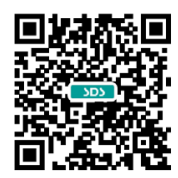




\section{INTRODUCTION}

With 25\%-30\%, fractures of the mandibular condylar process by now are most frequent among the different fracture locations of the mandible, with a growing incidence over the last decades ${ }^{[1-3]}$. Especially bicycle accidents are considered to contribute predominantly to this increase in Western Europe, accompanied by falls, faints and an overall increase of sports associated traumatic episodes ${ }^{[3,4]}$. In addition, the advent of easily accessible computerized tomography (CT) based trauma diagnostics has contributed significantly to a more frequent diagnosis especially of condylar head fractures ${ }^{[1,4]}$. Whereas this entity was considered to be rather rare with rates well below 5\% until the 70ies of the last century, condylar head fractures are by now diagnosed even more frequently than condylar neck fractures and now are second to fractures of the condylar base with a ratio of near to 2:3. Condylar fractures are reported to present mostly unilaterally, the ratio uni- $v s$. bilateral is currently given with around $4: 1^{[1]}$. Whereas the clinical relevance of condylar fractures goes undisputed with regard to potential adverse impact on temporomandibular joint (TMJ) function, posttraumatic malocclusion, and associated facial asymmetry pain and discomfort, optimum treatment of condylar fractures has long been counted among the most controversially discussed topics in oral and maxillofacial traumatology. This debate of surgical $v$ s. non-surgical treatment by now goes on without final consensus for already several decades. Whereas so called "conservative" (or better nonsurgical) treatment is based on a centuries old tradition, the advent of functionally stable osteosynthesis has progressively contributed to some paradigm shift since the turn of the century. Functionally stable osteosynthesis was introduced in the 1980ies, enabling for the first time to both perform an anatomically correct repositioning of the fragments and at the same time to efficiently mobilise the joint. In order to restore TMJ function early mobilisation by now may be considered as a prerogative for restoring normal TMJ functionality ${ }^{[5-7]}$. Miniplate based stable osteosynthesis thus allowed to overcome the unfavourable results of e.g., instable wire osteosynthesis and since the 1980ies step by step widened out the indications for surgical treatment also for the condylar process, paralleled by the aforementioned improvements in CT diagnostics ${ }^{[4,5]}$. More recently a continuous refinement of indication-specific osteosynthesis materials (OSMs), with a large variety of three-dimensional (3D)-plates specifically designed for the condylar base and neck and a diversity of resorbable materials for the condylar head being the latest traumatological developments in Oral and Maxillofacial Surgery, have contributed to transfer open reduction and rigid internal fixation (ORIF) of condylar base and lower neck fractures progressively into clinical routine. Parallelly, over the last decades, a series of new approaches was introduced, to cope with the specific demands for ORIF according to the different fracture locations, which following the recent consensus based Arbeitsgemeinschaft für Osteosynthesefragen (AO, Davos-Switzerland) comprehensive injury automatic classifier (AOCOIAC) classification ${ }^{[1]}$, are now subdivided into fractures of the condylar base, condylar neck and condylar head. By now even fractures of the condylar head, treated surgically by Rasse already in $1992^{[8]}$, have moved into a growing focus of surgical interest and in the meanwhile are far from being considered as "experimental", as dubbed still only a decade ago. According to recent consensus ${ }^{[9]}$ and based today on improved higher level of evidence ${ }^{[10,11]}$, ORIF of fractures of the condylar process may now be considered as the "golden standard" by a majority of surgeons for both displaced or dislocated condylar base and neck fractures in adults. Endoscopically assisted reduction and rigid fixation (EA-RIF) via transoral approaches is equally considered as an alternative for base fractures with lateral displacement ${ }^{[9,12]}$. Nevertheless, open reduction and rigid internal fixation (ORIF) in condylar fractures even today is far from being unanimously accepted as treatment of first choice. In Europe, or even more so worldwide, till today, there are no homogenous treatment concepts even in displaced or dislocated condylar fracture cases. Whereas among those surgeons, who by now routinely perform ORIF/EA-RIF in condylar process fractures, there is now a growing tendency to perform ORIF in condylar head fractures in adults and also severely displaced or dislocated fractures even in younger children with mixed dentition ${ }^{[13]}$, there is still a considerable number of surgeons who strongly favour closed reduction regimes ${ }^{[14,15]}$ or who - though basically in favour of ORIF - fail to cope with the full spectrum of condylar fracture osteosynthesis (e.g., very high fractures and head 
fractures, major fragmentation and multi-levelled fractures, endoscopically assisted surgery). The latter ones may still be considered to be a majority of active surgeons worldwide. Thus, at present, there is a noticeable partition regarding both concepts and/or skills and experience between oral and maxillofacial surgeons on a national and international scale regarding pro-surgery and traditional non-surgical and/or limited surgical concepts. Recently published, but fundamentally contradictory systematic reviews/meta-analyses add to this dilemma ${ }^{[10,14-16]}$. The present article is based on an invited lecture during the 24th Congress of the European Association for Cranio-Maxillo-Facial Surgery in Munich 2018 and wants to give an overview over current concepts from a predominantly surgical point of view, including some outlook and consideration regarding potential future developments in this highly specialised and controversial area.

\section{OPEN VS. CLOSED REDUCTION - INDICATIONS AND CONSIDERATIONS ON MANAGEMENT}

The aim of modern fracture treatment is to restore the patient's function and anatomy, preferentially both in as short time and with as little negative side-effects as possible ${ }^{[17]}$. In contrast to closed reduction, which at least in case of more pronounced displacement or dislocation usually will fail to restore pre-trauma anatomy, ORIF/EA-RIF allows for an anatomical correct repositioning. Basically, this will be feasible in almost all fractures depending, however, on the skill and experience of the surgeon in combination with availability of adequate hardware to perform a functionally stable osteosynthesis even in challenging localisations, such as thin condylar necks, fragmented head fractures, etc. As the traditional concepts of spontaneous repositioning of displaced/dislocated fractures after closed reduction have been shown to be "wishful thinking" and good remodelling can be expected in growing children until the early phase of the mixed dentation, only ${ }^{[9]}$, the previously unanimously accepted indications for closed reduction have been progressively narrowed down over the last decades. So closed reduction is still basically indicated in patients, who exhibit a reasonable (i.e., only slightly reduced) range of motion (especially regarding protrusion and laterotrusion within or close to normal physiological range of motion), no or minimal occlusal disorders as compared to the prefracture status (the assessment should follow the established rules of prosthodontic functional diagnostics to exclude forced muscular autocorrection), associated low discomfort or pain (i.e., which allows for early active training) and fractures in children (except for gross displacement or dislocation) up to the early stages of the mixed dentition ${ }^{[9]}$. So, basically, at least in isolated fracture cases the indication for closed $v s$. open reduction both in adults and older children (i.e., later stages of the mixed dentition) eventually is independent from fracture location and type of fracture ${ }^{[18]}$ and based on clinically based decision making rather than radiologically based assessment, only. Clinical experience has shown that e.g., in case of condylar head fractures (usually type " $m$ " according to the AO-classification proposal ${ }^{[1]}$ ) those patients without gross displacement/dislocation and pain will cope well via muscular adaptation and will show no or just minimal occlusal disorders when efficiently performing functional training ${ }^{[14,19,20]}$. Accordingly, the classical Zide and Kent ${ }^{[21]}$ surgical indication catalogue (bilateral fractures, soft tissue interposition, central dislocation, failed non-surgical therapy, edentulous patients not amenable to non-surgical therapy etc. ${ }^{[21]}$ ) have been narrowed down the crucial factor of ramus height instability (i.e., resulting in occlusal disorders and functional and possibly aesthetic asymmetry [Figure $1 \mathrm{~A}$ and $\mathrm{B}$ ] due to loss of vertical ramal/condylar height in case of displaced or rather frequently in dislocation fractures). Usually, a loss of vertical height more than $2-4 \mathrm{~mm}$ is associated with occlusal disorders ${ }^{[9,11]}$. As long as the head remains more or less properly positioned in the fossa, the proximal fragment may remain stable under mandibulo-maxillary fixation in patients with good occlusal support zones ${ }^{[22]}$. In case of instable occlusal support, the proximal fragment is prone to secondary displacement/dislocation due to the pull of the lateral pterygoid muscle ${ }^{[7,8,13,22]}$. Thus, in case of displaced fractures, the resulting fracture angulation of ramal stump versus proximal fragment is considered to be relevant first of all with regard to the resulting loss of vertical height, nevertheless potential long-term sequelae due to any dystopic head-to-fossa relations need to be taken into consideration, too ${ }^{[1]}$. Conversely, ORIF/EA-RIF is indicated if restoration of vertical dimension is required to maintain occlusion both with regard to the sagittal and transversal mandibular position ${ }^{[18,23]}$. Another crucial factor is prolonged 

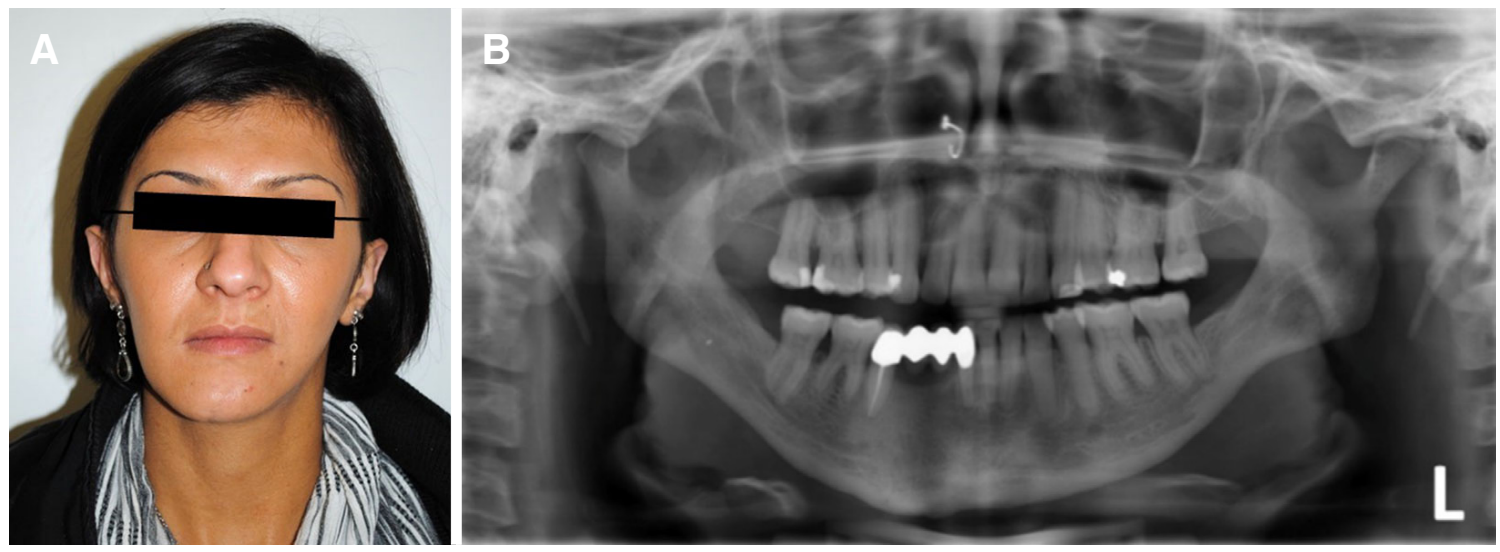

Figure 1. A: Facial asymmetry following non-surgical treatment of a condylar base fracture left hand side with loss of ramal height, however no impairment of range of motion, cantering of the occlusal plane due to occlusal compensation (levelling), so formally a good functional result, but bimaxillary surgery for skeletal deformity required; B: orthopantomogram 5 years post-trauma with shortening of the left ramus, orthotopic condylar position

(i.e., more than just for pain and edema reduction) immobilization of the TMJ, which is often required in closed reduction cases to maintain the proximal segment in a proper position for healing (especially contraction cases with loss of vertical height). Any immobilization, as often applied for 10-14 days in case of closed reduction via mandibulo-maxillary fixation (MMF) to improve occlusion, however, represents a major drawback, as it interferes with the basic principle of early immobilization in condylar fractures. Early mobilization has been proven to be beneficial, as e.g., hematomas will resolve quicker under movement whereas any immobilization over 4 weeks will lead to structural changes in the joint and diminished nutrition of the disc ${ }^{[22]}$. Thus, closed reduction - as long as immobilization procedures are required - will run a considerable risk of damaging the TMJ. In contrast, ORIF/EA-RIF offers both anatomical reduction and early mobilization of the TMJ, thus restoring physiological conditions. Accordingly, a recent metanalysis, here speaking in favour of surgery, points out "significant differences between ORIF and closed treatment regarding maximal interincisal opening, laterotrusive movement, protrusive movement, malocclusion, pain, and chin deviation on mouth opening $(P=0.001, P=0.001, P=0.001, P=0.001, P=0.001$, and $P=0.05$, respectively) showing ORIF being superior objectively and subjectively ${ }^{p\left[{ }^{[10]}\right.}$. Conversely, however, ORIF/EA-RIF will only be at an advantage, as long as the biomechanical standards of functionally stable osteosynthesis are sufficiently met and an early mobilization regime is observed. Or, to put it the other way round, any ORIF/EA-RIF or open reduction with a necessity for subsequent MMF or even more so if associated with hard and/or soft tissue damage will fail to give a competitive edge ${ }^{[17]}$. This especially applies for more challenging indications such as major fragmented fractures [Figure $2 \mathrm{~A}-\mathrm{D}$ ] and especially for not standard indications such as very high condylar neck and condylar head fractures.

According to, e.g., the author's personal experience with open reduction techniques gathered over for by now more than 25 years ORIF at least in standard indications (i.e., not considering fractures with direct TMJ involvement, major fragmented fractures, etc.), ORIF/EA-RIF almost invariably offers return to full functional recovery if properly done (i.e., restitutio ad integrum, which closed reduction will not be able to offer correspondingly). To meet these surgical standards, however, mistakes that are commonly made when starting with ORIF/EA-RIF procedures need to be eliminated when critically reviewing the literature. Thus, e.g., taking beginners' lack of surgical skills and experience - as unluckily often published in the literature - for certain complication rates into consideration (e.g., inadequate preauricular approach used for base fractures with result of elevated facial nerve palsies, cf. data according to the metanalyses by Al-Moraissi et al ${ }^{[24,25]}$ ) the conclusion of a recent systematic review speaking deliberately in favour of closed treatment becomes less astonishing, stating that "closed treatment appears to be a safe and appropriate 

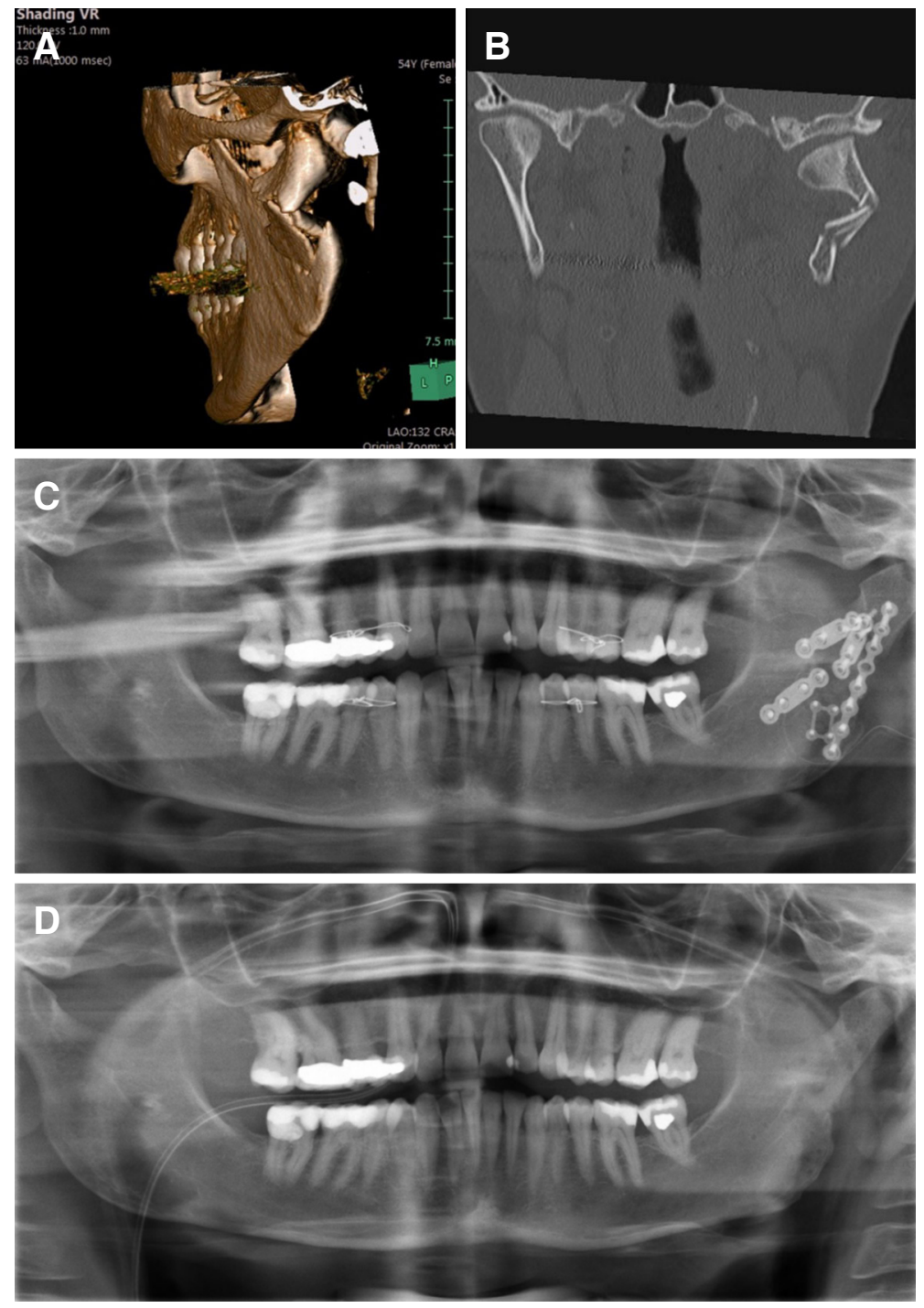

Figure 2. A: Three-dimensional computerized tomography (CT): major fragmented fracture of the ramus and condylar base left hand side with loss of vertical height, dystopic head to fossa relation; B: CT coronal view; C: orthopantomogram (OPT) postoperatively after open reduction and rigid internal fixation (combined transoral and extraoral approach); D: OPT after removal of osteosynthesis material showing restored anatomy. Full functional recovery, no occlusal disorders

modality for most unilateral condylar fractures. Although the open group in general showed similar outcomes, this treatment should be reserved for limited indications",[20]. The same review therefore "suggests that because of the high level of methodological variance in the relevant studies published to date, among other factors, there are currently no evidence-based conclusions or guidelines that can be formulated with regard to the most appropriate open treatment". Whereas the more experienced condylar surgeon will note with some frowning puzzlement this conspicuous contradictoriness of the currently available metaanlyses ${ }^{[10,14-16,20]}$, those surgeons less advanced in condylar traumatology may still find some convenient confirmation for the traditional non-surgical treatment concepts, thus widening the already existing gap between surgeons skilled in advanced ORIF of the condyle and those lacking experience ever more profoundly. Even the constantly repeated demand for future well designed randomized studies will not solve the dilemma, as by now surgically skilled condylar centers meet with serious problems to randomize their patients for a non-surgical group in which by now has become a standard surgical indication (in this context, e.g., cf. Smith and Pell ${ }^{[26]}$ dealing with randomized studies for parachuting). 

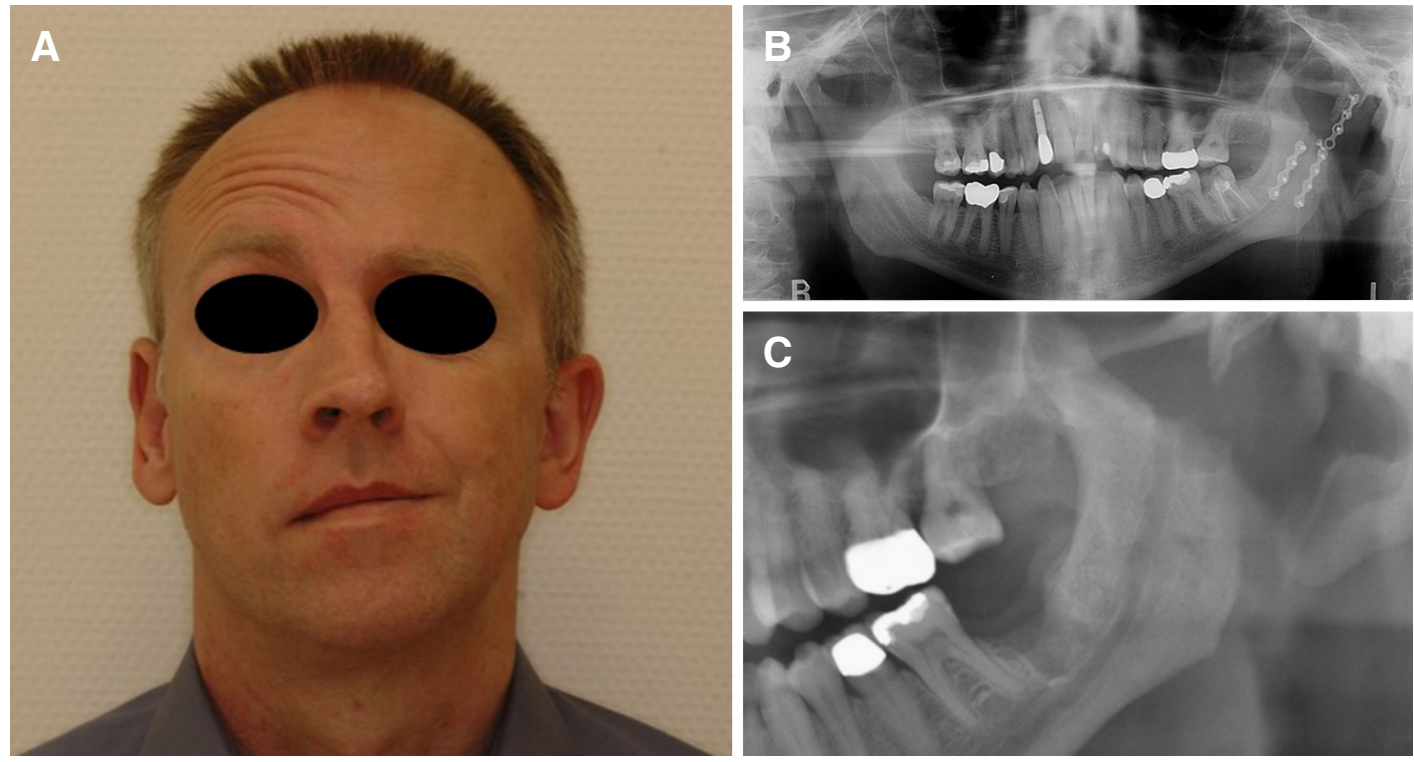

Figure 3. A: Permanent facial palsy left hand side and severe cantering of the occlusal plane after failed open reduction and rigid internal fixation of a combined condylar base to neck and ramus fracture left hand side; B: orthopantomogram showing loosening of osteosynthesis material after inadequate single miniplate fixation and development of pseudarthrosis, however no impairment of mouth opening; C: immobilized proximal fragment and fibrous pseudarthrosis with defect of the mandible requiring bimaxillary surgery and joint replacement

\section{SURGICAL TREATMENT - CONSIDERATIONS ON STATE OF THE ART MANAGEMENT}

Though ORIF/EA-RIF can offer superior results as outlined above, the other side of the coin - apart from a surgical intervention usually under general anesthesia - is its inherent capacity to provoke minor or even major complications [Figure 3A and B] if performed by less experienced hands. Although the biomechanical demands for stability in condylar process fractures have been published already some two decades ago $^{[27,28]}$ and thus should be commonplace by now, there are surgeons who rather inconvincibly perform osteosynthesis procedures which have been pointed out to be inefficient or prone to complications, such as single mini-plating for the condylar process, which may result in pseudarthrosis and in extreme cases even total loss of joint functionality [Figure $3 \mathrm{C}$ ]. Miniplating, though having been made public to be equally detrimental if applied for condylar head fractures ${ }^{[29,30]}$, nevertheless continues to be performed in quite a blue-eyed way by surgeons entering this very special indication even recently. As approaches play a major role to avoid complications and to achieve optimum biomechanical results in ORIF/EA-RIF of condylar fractures, the correct selection of a respective approach well suited for the different fracture levels is crucial. Fortunately, a recent meta-analysis by Al-Moraissi et al. ${ }^{[2,25]}$, which was in essence confirmed by a systematic review by Rozeboom et al. ${ }^{[15]}$, was able to show that facial nerve palsies are significantly correlated with some less suited approaches. According to these data, e.g., the peri-angular/high submandibular approach appears to be best suited for condylar base fractures up to the height of the sigmoid notch level with minimum facial nerve endangerment, in contrast to e.g., still often used preauricular approaches, which if applied for condylar base and lower condylar neck fractures - will be associated with an inadequate and therefore inacceptable risk of even permanent nerve palsies of around $4 \%{ }^{[25]}$. So basically, by now, sufficient information on a higher level of evidence should be at hand to choose an appropriate approach for the individual fracture level to avoid unnecessary hazard to the facial nerve, which is generally considered to be the most relevant counter-argument with regard to ORIF [Figure 3A]. Even EA-RIF, which is often erroneously propagated to be a rather risk-free alternative, may be well suited to avoid extraoral scars, but nevertheless runs some immanent risk of temporary facial nerve palsies, ranging between $3.3 \%, 4.6 \%$ and 7.7\% (transoral for lower fractures vs. transbuccal vs. EA-RIF osteosynthesis procedures ${ }^{[25]}$ ). Compared to temporary facial nerve palsy rates of the peri-angular/high submandibular approach of less than $1 \%{ }^{[24,25]}$ the 
transoral approaches are thus rather comparable to e.g., retroauricular approaches for the condylar head und upper condylar neck, here with rates between 3.3\% and $6.2 \%$ respectively ${ }^{[25]}$. So at present, intraoral approaches are rather considered as an alternative to extraoral approaches, when there is enough bone on both sides of the fracture lines to support a biomechanically stable osteosynthesis and may offer advantages and a quite safe procedure rather in selected cases ${ }^{[9,12,31-33]}$, which is usually the laterally displaced condylar base and conditionally the lower neck area. Surgeons using the preauricular approach for the upper condylar neck or condylar head should be able not to exceed temporary palsy rates of around $8 \%$. Surgeons using other approaches, such as, e.g., the classical retromandibular, anteroparotid or transparotid approaches should orient themselves at - and be qualified not to exceed - the respective temporary palsy rates, which by now are well published in the literature on a metanalysis level ${ }^{[15,24,25]}$. Otherwise - in case of not meeting these standards - the respective surgeon either needs to refine his technique or change to less risky approaches as described in the literature, instead. Though the risk of a permanent facial nerve palsy cannot be definitively excluded, this adverse event should be a very rare one if adequate technique and surgical skills are employed in this surgically demanding area. Or, to put it crudely, an inadequate selection of approaches and a conspicuous lack of surgical refinement and techniques - as unluckily often seen published in the literature by learners in the field of condylar fractures should not be taken as a thought-terminating cliché to discredit the beneficial options of ORIF/EA-RIF in favour of conventional closed reduction concepts.

\section{CURRENT TRENDS IN OSTEOSYNTHESIS MATERIALS}

Among surgeons practicing ORIF/EA-RIF of condylar fractures on a regular base, the focus of interest has by now shifted away from the old debate of "surgical vs. non-surgical therapy" towards more technically oriented questions, such as which approach is suited best for the different fracture locations ${ }^{[1,9,18,24,25,32]}$ and to the optimum choice of hardware to be applied with minimum complication rates. In this context there is now a running debate whether to use resorbable or standard titanium materials. In contrast to most other fracture locations of the facial skeleton, for which resorbable materials failed to establish as an alternative for titanium, the condylar process and especially the condylar head are currently heavily under discussion due to the risk for the facial nerve associated with OSM removal in this surgically demanding area. Though intriguing at first glance, the application of resorbable materials in the condylar region ${ }^{[34-38]}$, which of course allows to avoid a second surgery for OSM removal, nevertheless is associated with some considerable withdrawals from a biomechanical and tissue compatibility point of view. To achieve sufficient initial, but also secondary stability under functional training to restore physiological TMJ function, the material requires adequate volume and size to meet the high biomechanical demands required for this indication as pointed out above ${ }^{[17,27-29]}$. As e.g., poly-l-lactic acid/polyglycolic acid based resorbable materials, therefore, either need to be significantly bulkier than conventional titanium based materials [Figure 4A-D] to allow for early comparable loadings, the surgeon has to choose either to e.g., detach more soft tissues from the condylar fracture area or, alternatively, at least to partially immobilize a then less stable resorbable osteosynthesis ${ }^{[38]}$. Prolonged immobilization, especially in fractures directly involving the TMJ, on the other hand, has been identified as a major factor to promote intraarticular adhesions and soft tissue scarifications ${ }^{[7,22]}$. So some of the fundamental advantages of resorbable materials are thwarted either by a comparative increase in soft tissue trauma not only vis-a-vis a prolonged immobilization, a potentially more invasive approach or a modified (viz. extended) osteosynthesis design, but also towards potential adverse soft tissue reactions due to a prolonged acid degradation of the material itself, which, in turn, may affect long-term joint mobility with regard to a scarification of muscles and/or intra- or periarticular soft tissue structures ${ }^{[6,17]}$. This especially applies for fractures directly involving intraarticular structures, which have been shown to be significantly susceptible to the kind of osteosynthesis applied ${ }^{[7,30]}$. Though at present - aiming first of all at the idea to avoid osteosynthesis removal - recently some authors strongly advocate resorbable materials for condylar head fractures ${ }^{[34-37,39]}$, the author of the present article advises to apply resorbable material with some caution as long as these materials have not been proven to be at least equal in intermediate to long term results as compared to the functional and quality of life data by now available 

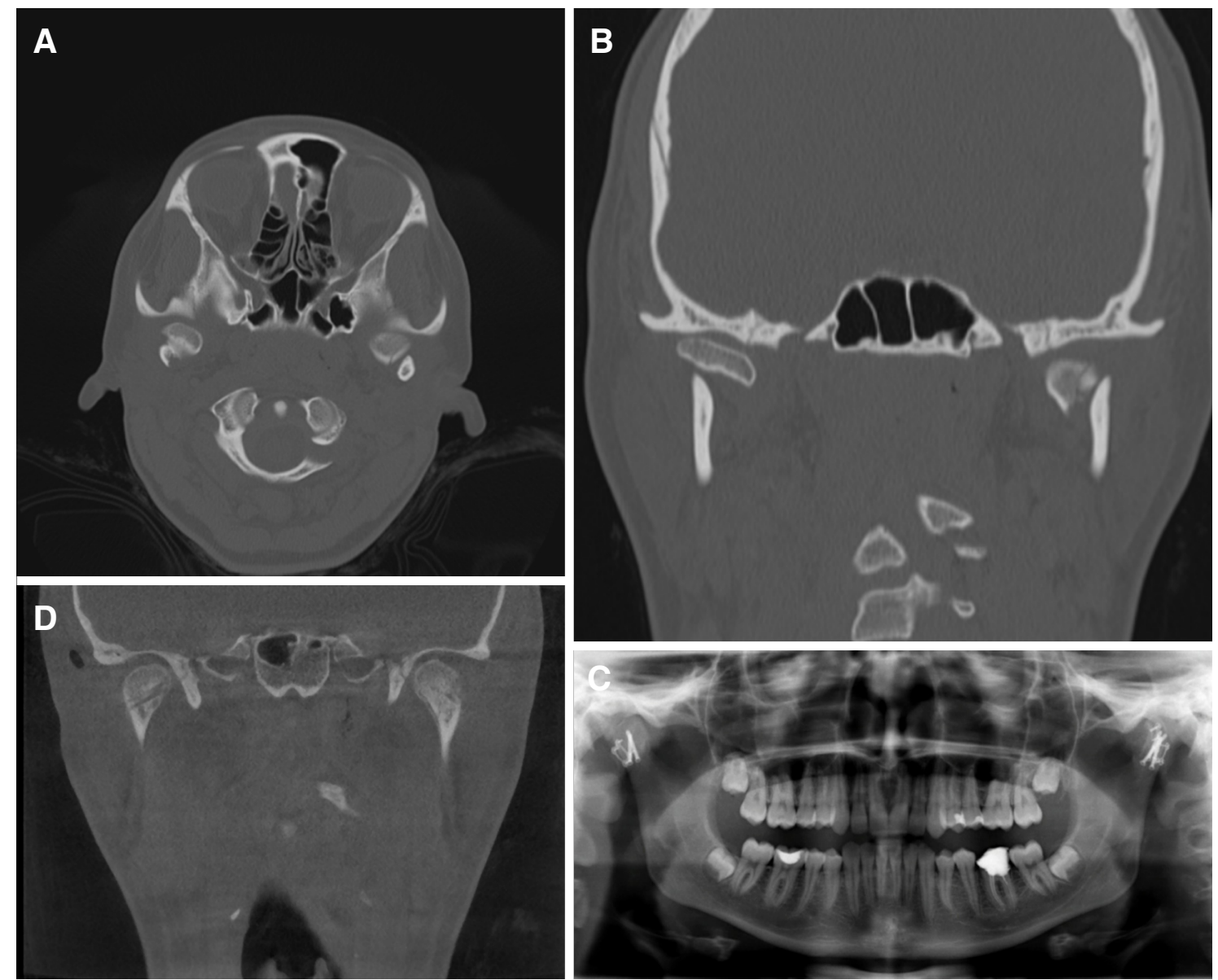

Figure 4. A: Bilateral condylar head fracture type $\mathrm{p}^{[1]}$ with loss of vertical height and occlusal disorder, left hand side with fragmentation, axial view; B: coronal view; C: orthopantomogram after osteosynthesis with positional screws and additional microplates to cope with fragmentation; D: condylar heads after removal of osteosynthesis material 4 months post-operatively, with vertical height restored to normal anatomy in cone beam computerized tomography coronal view, former screw positions still visible

for titanium materials ${ }^{[6,29]}$. In this context, however, it needs to be stressed that also titanium osteosynthesis, in spite of basically superior tissue compatibility, must be taken into consideration as a potential cause for long-term mechanical tissue irritation, which may lead to serious adverse side effects. This not only applies for failures of the osteosynthesis, which may lead to severe resorption and pseudarthrosis [Figure 3A-C], but also for basically well stabilized cases. According to the author's experience, condylar head fractures undergo a considerable remodeling process after stabilization, which may be explained, e.g., by stress shielding effects, thus leading to exposure of threads [Figure 5] in even as short a time as 3-4 months. As a consequence, several cases of scarification of the intra- and periarticular soft tissues leading to some even severe resorption of the condylar head have been noticed. This material induced interference has similar effects like fracture induced scarifications, leading to disc immobilisation and limitations ${ }^{[6,7]}$, which may induce osteoarthritis [Figure 6A and B]. This effect, in analogy, should of course be expected in case of any resorbable materials, as well. As a consequence, the author therefore strongly advocates an early removal of osteosynthesis material in case of titanium material, last but not least to prevent any late adverse sequelae such as osteoarthrosis. According to the author's experiences, removal of titanium material to prevent osseointegration of the screws, therefore, is considered to be mandatory ${ }^{[17]}$. Though, as a consequence, the idea of a material not requiring removal but offering biomechanical properties comparable to titanium remains intriguing especially for the condylar head area (e.g., magnesium based screws ${ }^{[39]}$ ), the multifold different recently published resorbable alternatives ${ }^{[34-39]}$ to titanium based systems still need to be fully assessed on a larger scale plus on a long-term base. As already pointed out above, any newly introduced 


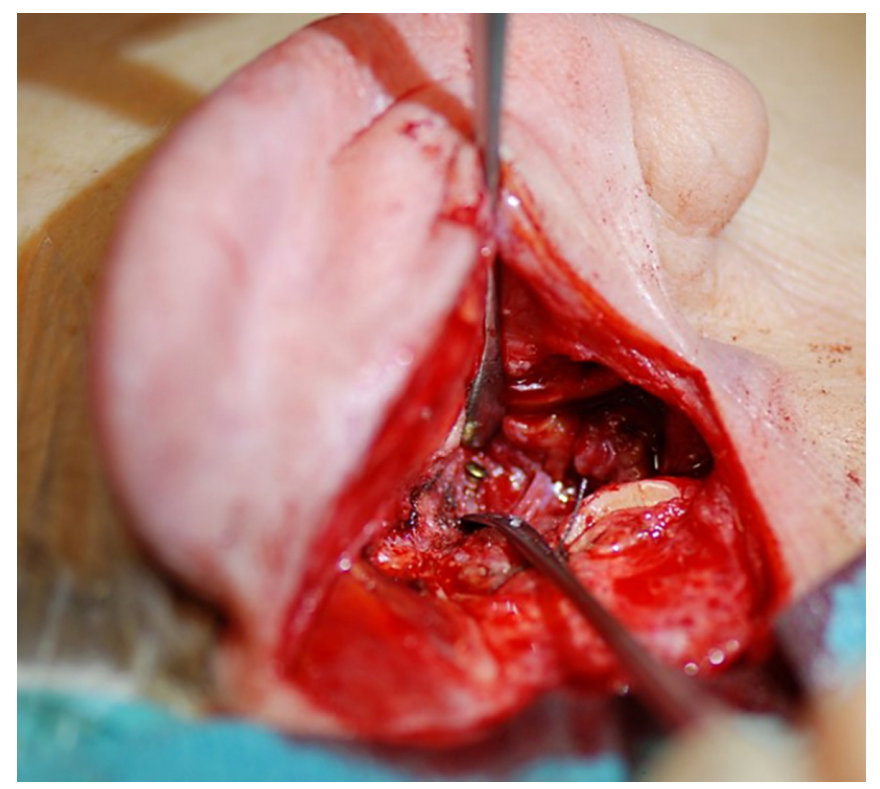

Figure 5. Resorption around a positional screw at removal of osteosynthesis material 3 months after open reduction and rigid internal fixation via retroauricular approach, most probably due to stress shielding

material will have to meet the standards with regard to stability, TMJ functionality and short to long-term complication rates as established, e.g., for titanium ${ }^{[6,17,29,30]}$.

\section{THE PARETO PRINCIPLE IN CONDYLAR FRACTURE TREATMENT}

According to the Pareto principle [which is also widely known as the "80/20 rule" established by Vilfredo Federico Pareto (1848-1923)] for many events, roughly $80 \%$ of the effects come from $20 \%$ of the causes, or to put it the other way round, we have to put $80 \%$ of our efforts to reach those $20 \%$ of the patients, who will not be managed fairly by the standard treatment protocols. This Pareto principle may therefore be also profoundly valid for condylar fractures as far as, e.g., fractures with major fragmentation, especially in the condylar head and neck area are concerned. Whereas standard cases including minor fragmentation in condylar base fractures in general do not represent any obstacles to a stable osteosynthesis and - as far as some fundamental biomechanical and surgical principles as pointed out above are observed - usually should yield good functional results with a low complication and morbidity rate (i.e., consuming $20 \%$ of our efforts according to the Pareto principle). On the other hand, the situation for e.g., multilevel compression fractures including the neck and head area and/or condylar head fractures with major fragmentation ${ }^{[1]}$ is a completely different one. Not for nothing some two decades ago these fractures were still considered "not to be amenable to safe and stable osteosynthesis ${ }^{\text {s[40] }}$ or at least "to be still experimental" according to a then valid consensus conference ${ }^{[41]}$. Even though by now an increasing number of surgeons are currently dealing with condylar head fractures, a great part either excludes or conspicuously do report on how to handle with cases with major fragmentation ${ }^{[36,38,42-44]}$. Considering a percentage of up to $40 \%$ of fragmented cases in condylar head fractures (around 30\% minor fragmented and some 10\% major fragmented cases in the author's collective comprising to date more than 360 head fractures ${ }^{[17]}$ ), this would mean that the surgeons may either just do selected cases (i.e., the "easier ones"), or will not routinely perform a sufficient osteosynthesis and/or abandon the procedure to perform e.g., a so-called debridement surgery. The latter one represents, at least in the author's view, more or less a confession of total failure, as this will end up in a situation even worse than preoperatively, as it prevents the mal-positioned condylar fragment to heal vis-a-vis a still basically functioning disc forming a nearthrosis in the articular eminence area ${ }^{[7,8,10,44]}$. The alternative would be the use of less efficient types of osteosynthesis (e.g., plating procedures ${ }^{[30,35]}$ ) which then, in turn, will promote 

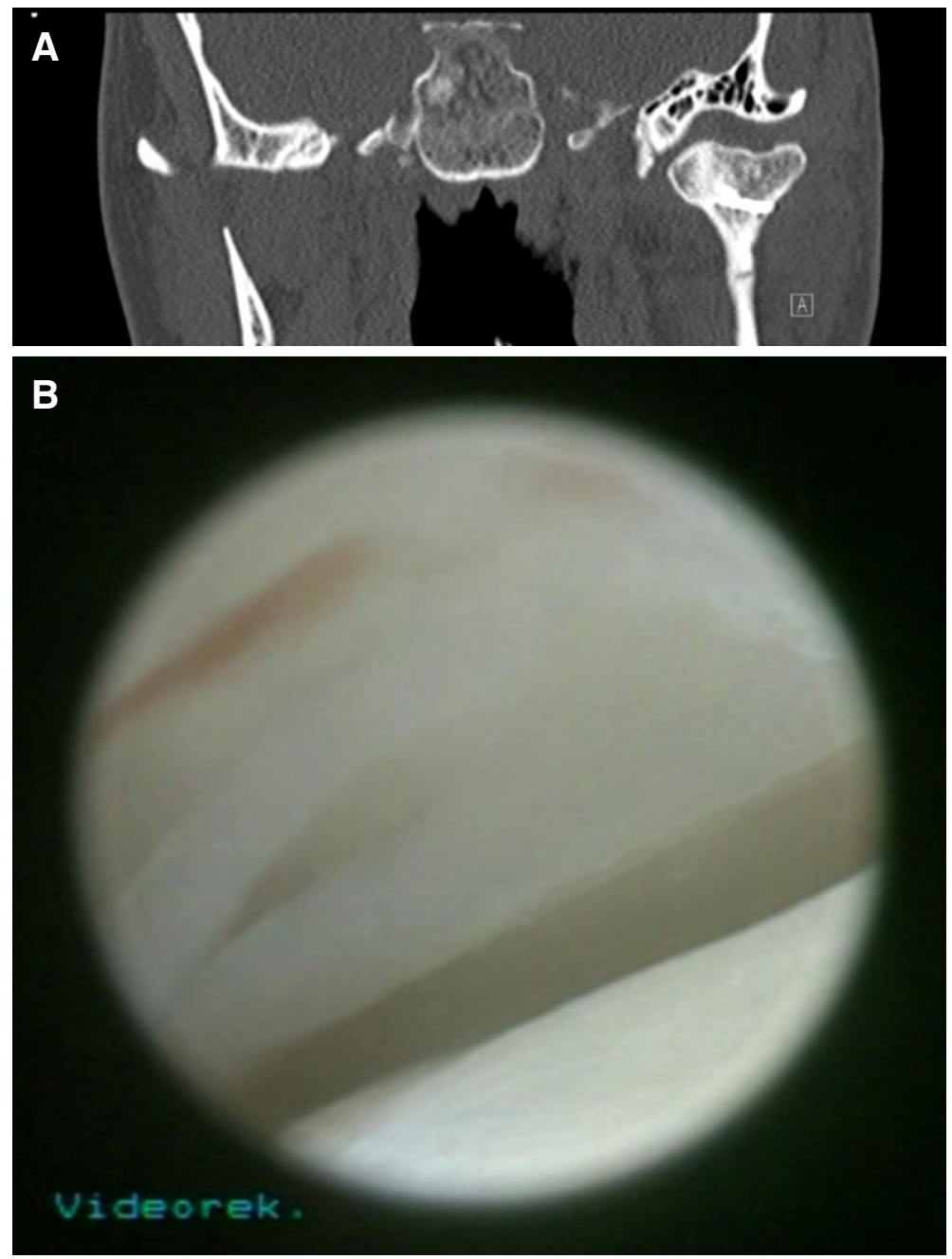

Figure 6. A: Osteoarthrosis with secondary resorptive defect of the articular surface after open reduction and rigid internal fixation of a condylar head fracture due to fusion of the disc in the lower joint space (cone beam computerized tomography coronal view); B: corresponding arthroscopy shows intraarticular scarring in the upper joint space resulting in limitations of range of motion

scarification, loosening of osteosynthesis material etc. due to interference with the intracapsular tissues and lack of stability ${ }^{[30]}$, and may end up in severe osteoarthrosis in the long run. Another major factor, which is often underestimated, is the clinically well known fact, that some trauma patients are not willing or capable to perform a consequent functional training. Patients' compliance, however, is mandatory in case of fractures directly involving the TMJ to prevent limitations due to scarification, regardless of any surgical or nonsurgical intervention. According to the Pareto principle, some $15 \%-20 \%$ of the patients - even if successfully repositioned and stabilized by ORIF - therefore will turn out to present with less than optimum possible results. Any reports of optimum healing rates well above $90 \%$ after ORIF, therefore, should be considered with some caution. In addition, unless properly done (i.e., minimizing intraarticular scarification), any intraarticular invasive procedure itself will be associated with a considerable risk of damaging the joint's functionality. So, again, refinement of surgical technique plays a paramount role regarding functional outcomes in this highly sensitive and surgically demanding area ${ }^{[17]}$. According to the author, when doing ORIF in condylar head fractures, some experience in functional TMJ surgery is therefore mandatory, as well. Considering the effect that some surgeons who are currently entering a new some of the pathways which have long been identified as less suited for condylar head fractures and which - at least to the author's experience - are prone to induce negative intermediate and long term sequelae ${ }^{[6]}$, the author by now had some "Road to Damascus experience". Thus, instead of strongly advocating ORIF in condylar head fractures 

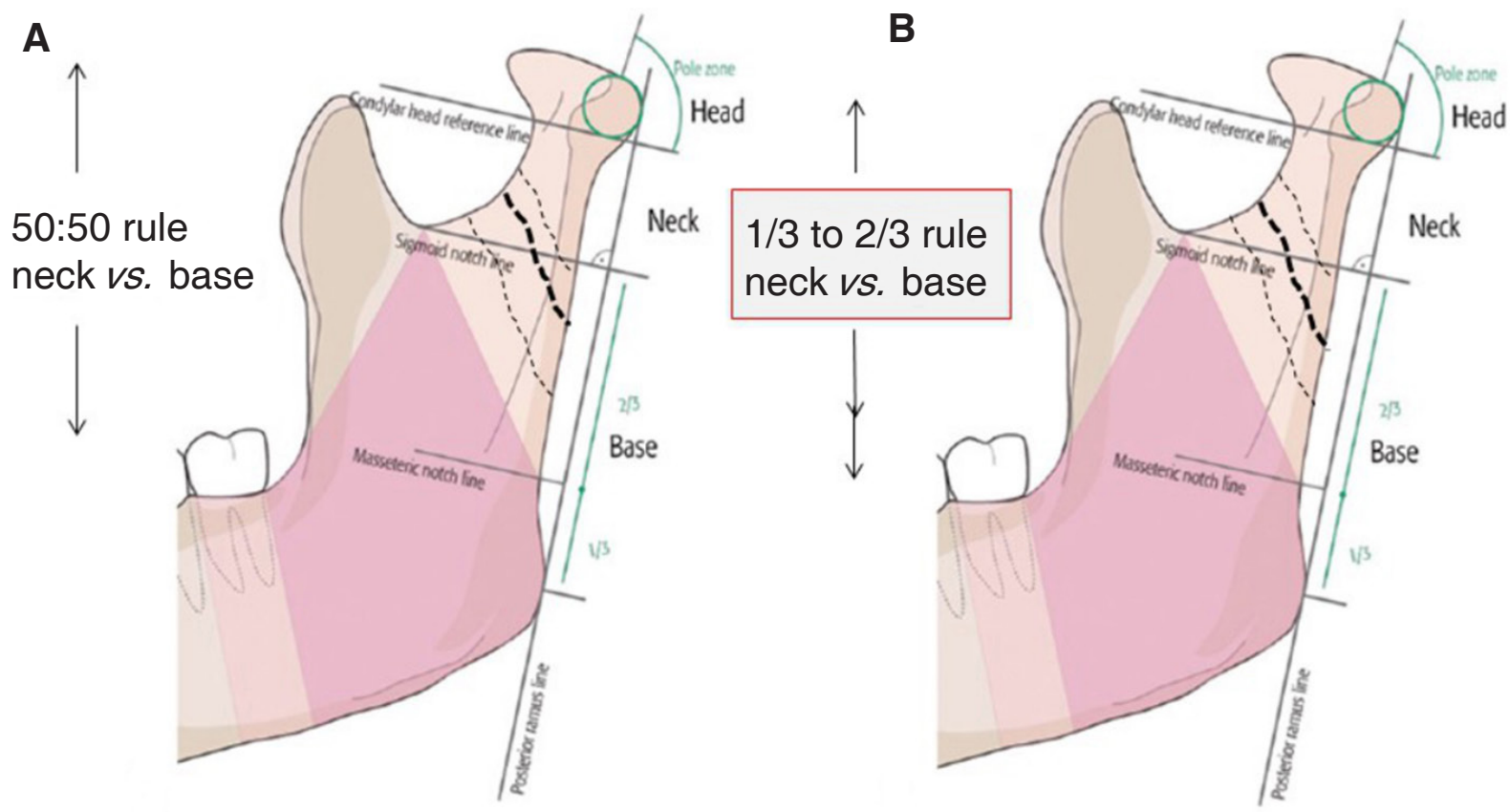

Figure 7. Classification of condylar process fractures according to Loukota et al. ${ }^{[45]}$ in 2005 left hand side according to the 50:50 rule; right hand side Arbeitsgemeinschaft für Osteosynthesefragen - classification proposal, now differentiating between base and neck fractures according to a $1 / 3$ to $2 / 3$ rule with regard to a reference line through the sigmoid notch perpendicular to the posterior border line (tangent). The head area is defined by a sphere comprising the lateral condylar pole zone ${ }^{[1]}$

for basically any displaced and /or dislocated head and also multifragmented condylar fractures, the author by now would rather recommend ORIF to be done not by the average surgeon, but only by those who are well familiar with both functional TMJ surgery and advanced condylar traumatology. Taking a look into the future, the Pareto principle again may apply for treatment of condylar head and major fragmented fractures: the majority (though hopefully progressively shrinking) will continue to be done non-surgically, whereas a minority (ideally augmenting paralleled by suited and adequate techniques) will be reserved for surgeons qualified to perform a both stable and as atraumatic as possible osteosynthesis. As a guiding principle, ORIF in condylar fractures should aim at reconstruction of both pre-trauma anatomy and pre-trauma function, or at least should be able to significantly minimize the trauma related negative sequelae with regard to TMJ functionality ${ }^{[17]}$.

\section{FUTURE PERSPECTIVES}

Regarding future developments in condylar traumatology, the author - who is without doubt strongly biased in favour of ORIF - personally envisions a future with ORIF in non-major fragmented condylar base and lower neck fractures to be performed on a routinely base, paralleled by a growing surgical experience with regard to well suited approaches, i.e., offering a truly low morbidity rate and a widely used application of biomechanically adequate osteosynthesis materials. This will hopefully take the wind out of the sails of the still strong supporters of non-surgical treatment with regard to relevant, but mostly avoidable complications associated with an invasive procedure ${ }^{[15,24]}$. This, at least according to the author's experience, can definitively be realized for the standard indications. As far as the more challenging indications - such as multilevel fractures, major fragmented base and neck fractures and fragmented head fractures - are concerned, the author strongly pleads for strictly considering the above mentioned principles, as well. Dealing with e.g., condylar head fractures, most of the fundamental anatomical and biomechanical principles have been described in detail by now already more than a decade ago ${ }^{[7,8,13,29,30]}$. To help avoid unnecessary complications - the author strongly recommends "not to reinvent the wheel", but rather to build up on pre-existing 
knowledge and sometimes hard-won experience of the protagonists, instead, in order not to discredit these advanced though still challenging techniques.

Another vision still to be realized in the future is establishing an internationally accepted classification of condylar fractures. The first successful steps have been done by Loukota et al. ${ }^{[45]}$ in 2005 developing the Strasbourg Osteosynthesis Research Group (SORG, Tuttlingen, Germany) initiated multi-center study ${ }^{[11]}$, which established the fundamental classification principles now being refined and basically integrated into the recent AO-classification proposal supported by SORG and the International Bone Research Association (IBRA, Basle, Switzerland) $^{[1]}$. The basic idea behind this classification proposal [Figure 7] is not only a landmark based classification allowing for unambiguous reproducibility, but also the concept that any classification needs to be based on validated consensus instead of individual appraisal. Finally, with the advent of 3D imaging by now easily available ${ }^{[1]}$, new techniques such as intraoperative image fusion and/or pre-planning with patient specific implants may find their way into condylar fracture osteosynthesis, e.g., facilitating and perhaps even improving surgical results, as long as the fundamental principles of soft tissue handling are regarded. Thus, condylar fracture treatment is by now on its way and looking forward to a promising surgical future, in accordance with Jules Verne: "Tout ce qui est dans la limite du possible doit être et sera accompli" (La Maison à Vapeur, 1880: "All that is within the limits of which is possible must and will be accomplished").

\section{DECLARATIONS}

Authors' contributions

Neff A solely contributed to the study.

Availability of data and materials

Not applicable.

\section{Financial support and sponsorship}

The lecture was given on invitation by EACMFS, with support for travelling and accommodation during the congress.

\section{Conflicts of interest}

The author has received remuneration as design surgeon for Medartis Basle-Ch, for scientific lectures for Karl Storz, Tuttlingen-D, as Head of the TMJ Section from SORG, Tuttlingen-D, as Head of the IBRA Educational Board from IBRA, Basle-Ch, as part of the AO -Classification Task Force from AO, Davos-Ch.

\section{Ethical approval and consent to participate}

Not applicable.

\section{Consent for publication}

Patients' consent for publication of pictures has been given, also for anonymized radiological cases consent for publication has been obtained.

\section{Copyright}

(c) The Author(s) 2019.

\section{REFERENCES}

1. Neff A, Cornelius CP, Rasse M, Dalla Torre D, Audigé L. The comprehensive AOCMF classification system: condylar process fractures level 3 tutorial. Craniomaxillofac Trauma Reconstr 2014;7:S044-58.

2. Niezen ET, Bos RRM, van Minnen B, Eckelt U, Tavassol F, et al. Fractures of the mandibular condyle: a comparison of patients, fractures 
and treatment characteristics between Groningen (The Netherlands) and Dresden (Germany). J Craniomaxillofac Surg 2018;46:1719-25.

3. Zachariades N, Mezitis M, Mourouzis C, Papadakis D, Spanou A. Fractures of the mandibular condyle: a review of 466 cases. Literature review, reflections on treatment and proposals. J Craniomaxillofac Surg 2006;34:421-32.

4. Rasse M. Recent developments in therapy of condylar fractures of the mandible. Mund Kiefer Gesichtschir 2000;4:69-87. (in German)

5. Kolk A, Neff A. Long-term results of ORIF of condylar head fractures of the mandible: a prospective 5-year follow-up study of smallfragment positional-screw osteosynthesis (SFPSO). J Craniomaxillofac Surg 2015;43:452-61.

6. Eckelt U, Loukota E, Loukota N. Condylar neck fracture treatment - historical review. In: Eckelt U, Loukota RA, editors. Fractures of the Mandibular Condyle - Approaches and Osteosynthesis. 2nd edition. Immenstadt: Eberl Medien GmbH; 2017. pp. 1-4.

7. Neff A, Kolk A, Horch HH. Position and mobility of the articular disk after surgical management of diacapitular and high condylar dislocation fractures of the temporomandibular joint. Mund Kiefer Gesichtschir 2000;4:111-7. (in German)

8. Kermer CH, Undt G, Rasse M. Surgical reduction and fixation of intracapsular condylar fractures. A follow up study. Int J Oral Maxillofac Surg 1998;27:191-4.

9. Neff A, Chossegros C, Blanc JL, Champsaur P, Cheynet F, et al. Position paper from the IBRA symposium on surgery of the head - the 2nd international symposium for condylar fracture osteosynthesis, Marseille, France 2012. J Craniomaxillofac Surg 2014;42:1234-49.

10. Al-Moraissi EA, Ellis E 3rd. Surgical treatment of adult mandibular condylar fractures provides better outcomes than closed treatment: a systematic review and meta-analysis. J Oral Maxillofac Surg 2015;73:482-93.

11. Eckelt U, Schneider M, Erasmus F, Gerlach KL, Kuhlisch E, et al. Open versus closed treatment of fractures of the mandibular condylar process-a prospective randomized multi-centre study. J Craniomaxillofac Surg 2006;34:306-14.

12. Haug RH, Brandt MT. Closed reduction, open reduction, and endoscopic assistance: current thoughts on the management of mandibular condyle fractures. Plast Reconstr Surg 2007;120:90S-102.

13. Neff A, Kolk A, Deppe H, Horch HH. New aspects for indications of surgical management of intra-articular and high temporomandibular dislocation fractures. Mund Kiefer Gesichtschir 1999;3:24-9. (in German)

14. Rozeboom AVJ, Dubois L, Bos RRM, Spijker R, de Lange J. Closed treatment of unilateral mandibular condyle fractures in adults: a systematic review. Int J Oral Maxillofac Surg 2017;46:456-64.

15. Rozeboom AVJ, Dubois L, Bos RRM, Spijker R, de Lange J. Open treatment of condylar fractures via extraoral approaches: a review of complications. J Craniomaxillofac Surg 2018;46:1232-40.

16. Rozeboom A, Dubois L, Bos R, Spijker R, de Lange J. Open treatment of unilateral mandibular condyle fractures in adults: a systematic review. Int J Oral Maxillofac Surg 2017;46:1257-66.

17. Neff A. Titanium screw osteosynthesis. In: Eckelt U, Loukota RA, editors. Fractures of the mandibular condyle - approaches and osteosynthesis. 2nd edition. Immenstadt: Eberl Medien GmbH; 2017. pp. 60-5.

18. Brandt MT, Haug R. Open versus closed reduction of adult mandibular condyle fractures: a review of the literature regarding the evolution of current thoughts on management. J Oral Maxillofac Surg 2003;61:1324-32.

19. Hlawitschka M, Eckelt U. Assessment of patients treated for intracapsular fractures of the mandibular condyle by closed techniques. J Oral Maxillofac Surg 2002;60:784-91.

20. Rozeboom AVJ, Klumpert LT, Koutris M, Dubois L, Speksnijder CM, et al. Clinical outcomes in the treatment of unilateral condylar fractures: a cross-sectional study. Int J Oral Maxillofac Surg 2018;47:1132-7.

21. Zide MF, Kent JN. Indications for open reduction of mandibular condyle fractures. J Oral Maxillofac Surg 1983;41:89-98.

22. Rasse M. Clinical experiences and evidence based results. In: Eckelt U, Loukota RA, editors. Fractures of the mandibular condyle approaches and osteosynthesis. 2nd edition. Immenstadt: Eberl Medien GmbH; 2017. pp. 5-9.

23. Zide MF. Outcomes of open versus closed treatment of mandibular subcondylar fractures (discussion). J Oral Maxillofac Surg 2001;59:375.

24. Al-Moraissi EA, Ellis E, Neff A. Does encountering the facial nerve during surgical management of mandibular condylar process fractures increase the risk of facial nerve weakness? A systematic review and meta-regression analysis. J Craniomaxillofac Surg 2018;46:1223-31.

25. Al-Moraissi EA, Louvrier A, Colletti G, Wolford LM, Biglioli F, et al. Does the surgical approach for treating mandibular condylar fractures affect the rate of seventh cranial nerve injuries? A systematic review and meta-analysis based on a new classification for surgical approaches. J Craniomaxillofac Surg 2018;46:398-412.

26. Smith GC, Pell JP. Parachute use to prevent death and major trauma related to gravitational challenge: systematic review of randomised controlled trials. BMJ 2003;327:1459-61.

27. Choi BH, Kim KN, Kim HJ, Kim MK. Evaluation of condylar neck fracture plating techniques. Int J Oral MaxillofacSurg 1999;27:109-12.

28. Meyer C, Kahn JL, Boutemi P, Wilk A. Photoelastic analysis of bone deformation in the region of the mandibular condyle during mastication. J Craniomaxillofac Surg 2002;30:160-9.

29. Neff A, Mühlberger G, Karoglan M, Kolk A, Koch S, et al. Stability of osteosyntheses for condylar head fractures in the clinic and biomechanical simulation. Mund Kiefer Gesichtschir 2004;8:63-74. (in German)

30. Neff A, Kolk A, Meschke F, Deppe H, Horch HH. Small fragment screws vs. plate osteosynthesis in condylar head fractures. Mund Kiefer Gesichtschir 2005;9:80-8. (in German)

31. Aziz SR, Ziccardi VB. Endoscopically assisted management of mandibular condylar fractures. Atlas Oral Maxillofac Surg Clin North Am 2009;17:71-4.

32. Haug R, Brandt MT. Traditional versus endoscope-assisted open reduction with rigid internal fixation (ORIF) of adult mandibular condyle fractures: a review of the literature regarding the evolution of current thoughts on management. J Oral Maxillofac Surg 2004;61:1272-9.

33. Schmelzeisen R, Cienfuegos-Monroy R, Schön R, Chen CT, Cunningham L Jr, et al. Patient benefit from endoscopically assisted fixation of condylar neck fractures - a randomized controlled trial. Oral Maxillofac Surg 2009;67:147-58.

34. Abdel-Galil K, Loukota R. Fixation of comminuted diacapitular fractures of the mandibular condyle with ultrasound-activated resorbable pins. Br J Oral Maxillofac Surg 2008;46:482-4. 
35. Landes C. Resorbable Osteosynthesis of condylar neck and head fractures. In: Eckelt U, Loukota RA, editors. Fractures of the mandibular condyle - approaches and osteosynthesis. 2nd edition. Immenstadt: Eberl Medien GmbH; 2017. pp. 69-81.

36. McLeod NM, Saeed NR. Treatment of fractures of the mandibular condylar head with ultrasound-activated resorbable pins: early clinical experience. J Oral Maxillofac Surg 2016;54:872-7.

37. Schneider M, Eckelt U, Reitemeier B, Meissner H, Richter G, et al. Stability of fixation of diacapitular fractures of the mandibular condylar process by ultrasound-aided resorbable pins (SonicWeld Rx® System) in pigs. Br J Oral Maxillofac Surg 2011;49:297-301.

38. Umstadt HE, Ellers M, Müller HH, Austermann KH. Functional reconstruction of the TM joint in cases of severely displaced fractures and fracture dislocation. J Craniomaxillofac Surg 2000;28:97-105.

39. Leonhardt H, Franke A, McLeod NMH, Lauer G, Nowak A. Fixation of fractures of the condylar head of the mandible with a new magnesium-alloy biodegradable cannulated headless bone screw. Br J Oral Maxillofac Surg 2017;55:623-5.

40. Baker AW, McMahon J, Moos KF. Current consensus on the management of fractures of the mandibular condyle. A method by questionnaire. Int J Oral Maxillofac Surg 1998;27:258-66.

41. Bos RR, Ward Booth RP, de Bont LG. Mandibular condyle fractures: a consensus. Br J Oral Maxillofac Surg 1999;37:87-9.

42. He D, Yang C, Chen M, Jiang B, Wang B. Intracapsular condylar fracture of the mandible: our classification and open treatment experience. J Oral Maxillofac Surg 2009;67:1672-9.

43. Smolka W, Cornelius CP, Lechler C. Resorption behaviour of the articular surface dome and functional outcome after open reduction and internal fixation of mandibular condylar head fractures using small-fragment positional screws. J Craniomaxillofac Surg 2018;46:1953-9.

44. Zheng J, Zhang S, Yang C, Abdelrehem A, He D, et al. Assessment of magnetic resonance images of displacement of the disc of the temporomandibular joint in different types of condylar fracture. Br J Oral Maxillofac Surg 2016;54:74-9.

45. Loukota RA, Eckelt U, De Bont L, Rasse M. Subclassification of fractures of the condylar process of the mandible. Br J Oral Maxillofac Surg 2005;43:72-3. 